\title{
KRULL DIMENSION AND INVERTIBLE IDEALS IN NOETHERIAN RINGS
}

\author{
by T. H. LENAGAN \\ (Received 18th September 1974)
}

In this note we consider the question: If $R$ is a right Noetherian ring and $I$ is an invertible ideal of $R$, how do the Krull dimensions of various modules, factor rings and over-rings of $R$, connected with $I$, compare with the Krull dimension of $R$ ? This question is prompted by results in (5) and (6). In comparing the Krull dimension of the ring $R$ with that of the ring $R / I$, the best result would be that the Krull dimension of the ring $R$ is exactly one greater than that of the ring $R / I$. This result is not true in general; however, we see, in Theorem 2.4 , that if the invertible ideal is contained in the Jacobson radical the result holds. In the general case we find it is necessary to introduce an over-ring $T$ of $R$ generated by the inverse $I^{-1}$ of $I$. We then see that the Krull dimension of $R$ is the larger of two possibilities: (a) Krull dimension of $R / I$ plus one or $(b)$ Krull dimension of $T$. In order to prove this result we construct a strictly increasing map from the poset of right ideals of $R$ to the cartesian product of the poset of right ideals of $T$ with a poset of certain infinite sequences of right ideals of $R / I$.

For background knowledge of Krull dimension we refer the reader to (2) and (4). We shall assume that each ring has a unit and that modules are unital.

\section{Definitions and preliminary results}

We recall first the definition of the deviation of a poset (partially ordered set). In (4) the definition was given only for positive integers, but, following Krause (3), we allow any ordinal number.

Let $E$ be a poset; if $a, b \in E$, then $[a, b]=\{x \in E \mid a \leqq x \leqq b\}$ is again a poset. If $E$ is a discrete set then $\operatorname{dev} E=-1$. If $E$ is Artinian (that is, each decreasing sequence of distinct elements of $E$ is finite) then $\operatorname{dev} E=0$. Suppose that, for a given ordinal $\alpha$, all posets $F$ with $\operatorname{dev} F<\alpha$ are known; then we define $\operatorname{dev} E=\alpha$ if each decreasing sequence $a_{1}, a_{2}, \ldots$ of elements of $E$ such that $\operatorname{dev}\left[a_{i+1}, a_{i}\right] \nless \alpha$, for each $i$, is finite. If there is no ordinal $\alpha$ such that $\operatorname{dev} E=\alpha$ we say that $E$ has no deviation.

This definition is applied to $\mathscr{L}(M)$, the lattice of $R$-submodules of an $R$ module $M$. The Krull dimension of $M$, denoted by $|M|$, is defined by $|M|=\operatorname{dev}(\mathscr{L}(M))$. In particular, $|R|=\operatorname{dev}\left(\mathscr{L}\left(R_{R}\right)\right)$.

Proposition 1.1 (see (3)). If $M$ is a Noetherian module, then $M$ has a Krull dimension.

E.M.S.-20/1-F 
Next we mention some elementary results from (4) concerning deviation. If $E, F$ are posets with deviation then so is $E \times F$ and

$$
\operatorname{dev}(E \times F)=\sup (\operatorname{dev} E, \operatorname{dev} F) .
$$

If $E$ is a poset, we define $\operatorname{Cr}(E)$ to be the set of sequences $\left(e_{1}, e_{2}, \ldots\right)$ of elements of $E$ such that $e_{1} \leqq e_{2} \leqq \ldots$ and that the $e_{i}$ become constant for $i$ large enough. The set $\operatorname{Cr}(E)$ is a poset under the ordering $e \leqq f$ if and only if $e_{i} \leqq f_{i}$, for each $i$. In (4) it is shown that $\operatorname{dev}(\operatorname{Cr}(E))=\operatorname{dev} E+1$. Let $E, F$ be posets with deviation, a map $f: E \rightarrow F$ is said to be increasing if, for all $x, y \in E, x \leqq y$ implies that $f(x) \leqq f(y)$. If $x<y$ implies that $f(x)<f(y)$, we say that the map is strictly increasing. If there is a strictly increasing map from $E$ to $F$ it is easy to see that $\operatorname{dev} E \leqq \operatorname{dev} F$.

An ideal $I$ of a ring $R$ is said to be an invertible ideal if there exists a subset, $I^{-1}$, of some over-ring $Q$ of $R$ with $1_{Q}=1_{R}$, such that $I^{-1}=I^{-1} I=R$.

Lemma 1.2. If $I$ is an invertible ideal of a ring $R$, with Krull dimension, then $|R / I|<|R|$.

Proof. First note that the structure of $R / I$ as an $R / I$-module is the same as its $R$-module structure. Suppose that $J, K$ are right ideals of $R$ with $I \leqq J<K \leqq R$, then $I^{n+1} \leqq J I^{n}<K I^{n} \leqq I^{n}$, and so we have a strictly increasing map from $\mathscr{L}(R / I)$ to $\mathscr{L}\left(I^{n} / I^{n+1}\right)$. Hence $\left|I^{n} / I^{n+1}\right| \geqq|R / I|$ and so the infinite descending chain of ideals $R>I>I^{2}>\ldots$ shows that $|R / I|<|R|$.

If $I$ is an invertible ideal of a ring $R$, it is easy to see that the ideals $I^{n}, n=2,3, \ldots$, are also invertible and that $T=\bigcup_{n=1}^{\infty} I^{-n}$ is an over-ring of $R$.

Proposition 1.3. If $R$ is a right Noetherian ring with invertible ideal I and $T=\bigcup_{n=1}^{\infty} I^{-n}$, then

(a) If $J$ is a right ideal of $T$, we have $J=(J \cap R) T$;

(b) $T$ is a right Noetherian ring;

(c) $|T| \leqq|R|$.

Proof. (a) If $j \in J$ then $j \in I^{-n}$, for some $n$, and therefore $j I^{n} \subseteq J \cap R$. Thus $j \in j I^{n} . I^{-n} \subseteq(J \cap R) T$.

(b) Obvious.

(c) The map $J \rightarrow J \cap R$ gives a strictly increasing map from $\mathscr{L}(T)$ to $\mathscr{L}(R)$; thus $|T| \leqq|R|$.

Proposition 1.4 (1, Lemma 1.1). If I is an invertible ideal of a right Noetherian ring $R$ and $E$ is a right ideal of $R$, there exists an integer $n=n(E)$, such that $E \cap I^{n} \subseteq E I$. 
2.

Throughout this section the following notation is used; $R$ is a right Noetherian ring; $I$ is an invertible ideal of $R ; T=\bigcup_{n=1}^{\infty} I^{-n} ; K, L$ are right ideals of $R$ and $J=J(R)$ is the Jacobson radical of $R$.

We would like to make the inequalities of Lemma 1.2 and Proposition $1.3(c)$ into some kind of equalities. This seems to be impossible in the general case. However, we are able to show that the two inequalities are closely connected. Recalling Kaplansky's comment on invertible ideals (a sort of poor man's principal ideal) and remembering the Principal Ideal Theorem, one might hope to prove that $|R|=|R / I|+1$. This is obviously impossible if $|R|$ is a limit ordinal and is also not necessarily true when rings of finite Krull dimension are investigated. What we are able to show is that if this equality does not hold then the rings $R$ and $T$ have the same Krull dimension. The key result is the following simple lemma.

Lemma 2.1. Let $L \subseteq K$ be right ideals of $R$ such that

(a) $K I \subseteq L$;

(b) $L+I^{n}=K+I^{n}$, for $n=1,2, \ldots$

Then $L=K$.

Proof. By Proposition 1.4, there is an integer $n$ such that $K \cap I^{n} \subseteq K I \subseteq L$. Thus $K \cap I^{n}=L \cap I^{n}$.

Let $X=K+I^{n}=L+I^{n}$ and consider the module $X / L$.

We see that

$$
X / L=\left(L+I^{n}\right) / L \cong I^{n} /\left(L \cap I^{n}\right)=I^{n} /\left(K \cap I^{n}\right) \cong\left(K+I^{n}\right) / K=X / K .
$$

If $L \neq K$, we have shown that $X / L$ is isomorphic to a proper factor module of itself. Since $X / L$ is Noetherian, this is obviously impossible.

Lemma 2.2. Let $L \subseteq K$ be right ideals of $R$ and suppose that

$$
\left(L \cap I^{n}\right)+I^{n+1}=\left(K \cap I^{n}\right)+I^{n+1}, \text { for } n=0,1,2, \ldots
$$

Then $L+I^{n}=K+I^{n}$, for $n=0,1,2, \ldots$

Proof. This is an easy inductive proof.

Our next objective is to construct an increasing map from $\mathscr{L}(R)$ to $\operatorname{Cr}(\mathscr{L}(R / I))$. The map we are interested in is the map $\theta$ where $\theta(K)$ is

$$
\left((K+I) / I,\left((K \cap I) I^{-1}+I\right) / I, \ldots,\left(\left(K \cap I^{n}\right) I^{-n}+I\right) / I, \ldots\right) .
$$

We only need to verify that $\theta(K) \in \operatorname{Cr}(\mathscr{L}(R / I))$, since the fact that $\theta$ is increasing is trivial. Now $\left(K \cap I^{n}\right) I^{-n} \subseteq R$, for each $n=1,2, \ldots$, and

$$
\left(K \cap I^{n}\right) I^{-n}=\left(K \cap I^{n}\right) I . I^{-n-1} \subseteq\left(K \cap I^{n+1}\right) I^{-n-1} .
$$

Therefore, since $R$ is right Noetherian, $\theta(K) \in \operatorname{Cr}(\mathscr{L}(R / I))$. 
Theorem 2.3.

$$
|R|=\max \{|T|,|R / I|+1\} .
$$

Proof. If we are able to construct a strictly increasing map from $\mathscr{L}(R)$ to $\mathscr{L}(T) \times \operatorname{Cr}(\mathscr{L}(R / I))$, then

$$
\begin{aligned}
|R| & \leqq \operatorname{dev}(\mathscr{L}(T) \times \operatorname{Cr}(\mathscr{L}(R / I))) \\
& =\max (|T|,|R / I|+1),
\end{aligned}
$$

and the result follows from Lemma 1.2 and Proposition $1.3(c)$.

The map we use is $\Phi$ where $\Phi(K)=(K T, \theta(K))$, and $\theta$ is the map defined above. This is obviously an increasing map, we need only check that it is strict. Suppose that $L \subsetneq K$ are right ideals of $R$ such that $\Phi(L)=\Phi(K)$. Using the Noetherian property, we may assume that $K / L$ is a simple module. From the fact that $\theta(L)=\theta(K)$ we deduce, using Lemma 2.2, that $L+I^{n}=K+I^{n}$, for each $n$. Now $K \subseteq K T=L T$, and so, if $k$ generates $K$ modulo $L, k I^{n} \subseteq L$, for some integer $n$. Since $K / L$ is simple, we see that $k I \subseteq L$ and thus $K I \subseteq L$. Hence the conditions of Lemma 2.1 are satisfied and we deduce that $L=K$. Therefore $\Phi$ is a strictly increasing map.

We shall see that either of the two equalities in this theorem may fail. However, in one case, we can be more specific.

Theorem 2.4 (cf. 6, Theorem 1.9). If I is contained in the Jacobson radical of $R$ then $|R|=|R / I|+1$.

Proof. The proof is similar to that of the previous theorem except that we need only consider the map $\theta$, which has already been defined. The fact that this is a strictly increasing map follows easily from the next lemma.

Lemma 2.5. If $I \subseteq J(R)$ then $E=\bigcap_{n=1}^{\infty}\left(E+I^{n}\right)$, for all right ideals $E$ of $R$.

Proof. Suppose that the result is false and choose a right ideal $E$ maximal among those for which the result fails. Then $E^{\prime}=\bigcap_{n=1}^{\infty}\left(E+I^{n}\right)>E$. Now, if $K$ is any right ideal properly containing $E$, it is obvious that $E^{\prime} \leqq K^{\prime}=K$. Hence $E^{\prime} / E$ is a simple module and so $\left(E^{\prime} / E\right) J=0$, from which it follows that $E^{\prime} I \subseteq E$. However, by Proposition 1.4, there is an integer $s$ with

$$
E^{\prime} \cap I^{s} \subseteq E^{\prime} I \subseteq E \text {. }
$$

Therefore $E=E+\left(E^{\prime} \cap I^{s}\right)=E^{\prime} \cap\left(E+I^{s}\right)=E^{\prime}$, which is a contradiction.

In the case of a ring with finite Krull dimension we are able to give another proof of Theorem 2.4 , following directly from Theorem 2.3 . We need the following result from (4). 
Lemma 2.6. Let $E, F$ be posets with finite deviation and suppose that $f: E \rightarrow F$ is an increasing map. If there are non-negative integers $n, r$ such that $\operatorname{dev}[a, b] \geqq n$ implies that $\operatorname{dev}[f(a), f(b)] \geqq r$, then

$$
\operatorname{dev} E+r \leqq \sup (n+r, \operatorname{dev} F+n) \text {. }
$$

In particular, if $a<b$ implies that $\operatorname{dev}[f(a), f(b)] \geqq r$, then $\operatorname{dev} E+r \leqq \operatorname{dev} F$.

Suppose now that $R$ is a ring with finite Krull dimension and that $I$ is an invertible ideal contained in $J(R)$, the Jacobson radical of $R$. If $L \leqq K$ are right ideals such that $K / L$ is Artinian then some power of $I$ will annihilate $K / L$ and thus $L T=K T$. With this in mind, we see that the map $f: \mathscr{L}(T) \rightarrow \mathscr{L}(R)$, given by $f(A)=A \cap R$, has the property that $A<B$ implies $|(B \cap R) /(A \cap R)| \geqq 1$. Therefore, from the lemma, $|T|+1 \leqq|R|$ and $|T|<|R|$. Hence, from Theorem 2.3, we conclude that $|R|=|R / I|+1$.

The reason for giving this alternative proof for a special case is that it suggests a generalisation of the Jacobson radical. A module $M$ is said to be $\alpha$-critical if $|M|=\alpha$ but $|M / N|<\alpha$, for each non-zero submodule $N$ of $M$. It is obvious that simple modules are exactly the 0-critical modules and so we can think of the Jacobson radical of $R$ as the intersection of the annihilators of the $\mathbf{0}$-critical modules. In this way we are led to define, for each positive integer $n$, $J_{n}(R)=\bigcap A$, where $A$ is the annihilator, in $R$, of a critical module whose Krull dimension is less than or equal to $\alpha$. The $J_{n}(R)$ are ideals of $R$ and $J(R)=J_{0}(R) \supseteq J_{1}(R) \supseteq \ldots$

Proposition 2.7. Let $R$ be a ring with finite Krull dimension and $I$ an invertible ideal of $R$ with $I \subseteq J_{n}(R)$, for some integer $n$. Then, if $T=\bigcup_{n=1}^{\infty} I^{-n}$, we have $|T|+n+1 \leqq|R|$.

Proof. The proof is similar to the reasoning above. We need only observe that if $L \leqq K$ are right ideals of $R$ with $|K / L| \leqq n$ then $L T=K T$.

The most obvious example of an invertible ideal is an ideal generated by a regular central or normalising element (an element $x$ is a normalising element if $x R=R x$ ). We shall say that $R$ is an $N$-ring if each proper ideal of every prime factor ring of $R$ contains a normalising element of the factor ring (right Noetherian polynomial identity rings satisfy this condition).

Theorem 2.8. Let $R$ be an $N$-ring and $J$ its Jacobson radical. Suppose that $|R / J|=\alpha$. Then $|R|=\alpha+n$, for some non-negative integer $n$.

Proof. Using the Noetherian condition we may assume, by way of a contradiction, that the result fails for $R$ but holds for proper factor rings of $R$, and that $|R|=\beta$. There is a prime ideal $P$ of $R$ with $|R / P|=\beta$. If $P \neq 0$, then the result holds in the ring $R / P$. In this case, if $K$ is the inverse image in $R$ of the Jacobson radical of $R / P$, we have that $\beta=|R / K|+n$, for some $n$. However, $J \subseteq K$ and so $|R / K| \leqq|R / J|$. Hence we see that $\alpha \leqq \beta \leqq \alpha+n$. Therefore 
we may assume that $R$ is a prime ring. If $J(R)=0$, then we are finished. Otherwise, choose $x \in J$, a normalising element. Then by Theorem 2.4, $|R|=|R / x R|+1$. Now $J / x R$ is the Jacobson radical of $R / x R$ and so, by assumption, $|R| x R|=| R / J \mid+n$, for some integer $n$. But then

a contradiction.

$$
|R|=|R / x R|+1=|R / J|+n+1,
$$

This last result is not true in general; see, for example, the rings of Jategaonkar (2, Ex. 10.3). As a corollary to this theorem, we mention a result of Small.

Corollary 2.9. If $R$ is a right Noetherian, semi-local polynomial identity ring then $R$ has finite Krull dimension.

Proof. If $J$ is the Jacobson radical of $R$, then $|R / J|=0$. Therefore, by the theorem, $|R|=0+n=n$, for some integer $n$.

We have seen that if $I$ is an invertible ideal contained in the Jacobson radical of $R$ then $|T|<|R|$. This occurs, for instance, if $I$ is the ideal generated by 2 in $R$, where $R$ is the ring of integers localised at the ideal $2 \mathbb{Z}$. In this case, $|R|=1$ and $|T|=0$; in fact, $T=Q$, the field of rational numbers.

In order to show that the other equality of Theorem 2.3 may fail we proceed as follows. Let $S$ be the ring of polynomials in commuting indeterminates $x, y$ and $z$ over a field. Let $R$ be the localisation of $S$ with respect to the complement of the set $x S \cup(y S+z S)$. Then $|R|=2$ and $|R / x R|=0$.

\section{REFERENCES}

(1) A. W. Chatters and S. M. GinN, Localisation in hereditary rings, J. Algebra 22 (1972), 82-88.

(2) R. Gordon and J. C. Robson, Krull dimension, Memoirs American Math. Soc. 133 (1973).

(3) G. Krause, On the Krull dimension of left Noetherian, left Matlis rings, Math. Z. 118 (1970), 207-214.

(4) R. Rentschler and P. Gabriel, Sur la dimension des anneaux et ensembles ordonnes, C.R. Acad. Sci. Paris 265 (1967), 712-715.

(5) P. F. Smith, On the dimension of group rings, Proc. London Math. Soc. 25 (1972), 288-302; Corrigendum, 27 (1973), 766-768.

(6) R. WALKer, Local rings and normalising sets of elements, Proc. London Math. Soc. 24 (1972), 27-45.

Mathematical Institute

UNIVERSITY OF EDINBURGH 